\title{
Incidência de conduta violenta e antissocial em população psiquiátrica forense
}

\author{
Incidence of violent and antisocial behavior in a forensic psychiatric population
}

\author{
Lisieux Elaine de Borba Telles ${ }^{1}$, Jorge O. Folino ${ }^{2}$, José G. V. Taborda ${ }^{3}$ \\ ${ }^{1}$ Mestre em Psiquiatria Forense, Universidade Nacional de La Plata (UNLP), La Plata, Argentina. Doutora, UNLP. Professora, Programa de Residência Médica em \\ Psiquiatria Forense, Universidade Federal de Ciências da Saúde de Porto Alegre (UFCSPA) e Hospital Psiquiátrico São Pedro (HPSP), Porto Alegre, RS. ${ }^{2}$ Doutor \\ em Medicina, UNLP. Professor titular de Psiquiatria, UNLP. ${ }^{3}$ Mestre e Doutor em Medicina: Clínica Médica, Universidade Federal do Rio Grande do Sul (UFRGS), \\ Porto Alegre, RS. Professor adjunto, Departamento de Clínica Médica, UFCSPA. Coordenador, Programa de Residência Médica em Psiquiatria Forense, UFCSPA. \\ Presidente, Section of Forensic Psychiatry, World Psychiatric Association.
}

Este artigo apresenta resultados da tese intitulada "Predicción de la conducta violenta en la población psiquiátrica forense a través de la HCR-20 y PCL-R", defendida no ano de 2009 junto à Universidade Nacional de La Plata, La Plata, Argentina. O estudo recebeu os prêmios Prof. Álvaro Rubim de Pinho, da Associação Brasileira de Psiquiatria (ABP), e Paulo Guedes, da Associação de Psiquiatria do Rio Grande do Sul (APRS), ambos em 2009.

\section{Resumo}

Introdução: As consequências de comportamentos violentos e antissociais de internos em hospitais psiquiátricos são graves e podem determinar prejuízo ao tratamento do agressor. A falta de conhecimento sobre a incidência desse fenômeno e suas características favorece sua perpetuação.

Objetivos: Apresentar uma revisão bibliográfica atualizada do tema, informar a incidência de conduta violenta e antissocial em uma população manicomial e descrever a implementação de um método de registro sistematizado desse fenômeno.

Método: A coorte foi selecionada dentro da população de pacientes do Instituto Psiquiátrico Forense Maurício Cardoso (IPFMC). Foi utilizada a Escala de Agressividade Declarada de Yudofsky (Overt Aggression Scale, OAS) e o Questionário de Seguimento de Tengström et al. Durante o período de seguimento de 1 ano, foram avaliados diariamente episódios de conduta violenta e antissocial, registrando-se os mesmos na escala e no questionário. A amostra em estudo foi composta de 68 pacientes homens selecionados de forma aleatória entre a população em cumprimento de medida de segurança no IPFMC.

Resultados: A incidência de comportamento violento/antissocial no período estudado foi de 200 episódios, envolvendo 50 pacientes. Houve predomínio de agressões e conduta antissocial durante o dia. As intervenções mais utilizadas foram manejo verbal, isolamento do paciente e aplicação de medicação intramuscular.

Conclusão: Foi possível, pela primeira vez, medir a ocorrência de conduta violenta e antissocial em uma população psiquiátrica forense.

Descritores: Transtornos mentais, violência, incidência, medição de risco, avaliação.

\begin{abstract}
Introduction: The consequences of violent and antisocial behaviors among psychiatric hospital inpatients are serious and may affect treatment of the aggressor. Lack of knowledge concerning the incidence of this phenomenon and its characteristics helps perpetuate the current scenario.

Objectives: To present an up-to-date literature review on the topic, to estimate the incidence of violent and antisocial behaviors in a mental hospital population, and to describe the implementation of a systematic method to record this phenomenon.

Method: A cohort was selected among the psychiatric inpatients at Maurício Cardoso Forensic Psychiatry Institute (Instituto Psiquiátrico Forense Maurício Cardoso, IPFMC). Yudofsky's Overt Aggression Scale (OAS) and Tengström et al.'s Follow-Up Questionnaire were used. During a 1-year follow-up period, episodes of violent and antisocial behavior were evaluated daily and recorded on both the scale and the questionnaire. The population sample included 68 male patients randomly selected among the committed inpatients at IPFMC.

Results: The incidence of violent/antisocial behavior along the period assessed was 200 episodes involving 50 patients. Aggressions and antisocial behavior occurred mainly in daytime. The interventions most commonly used were verbal management, patient isolation, and intramuscular medication.

Conclusion: For the first time, the occurrence of violent and antisocial behavior was measured in a forensic psychiatric population.
\end{abstract}

Keywords: Mental disorders, violence, incidence, risk assessment, evaluation.

\section{Correspondência:}

Lisieux Telles, Av. Caçapava, 537/402, CEP 90460-130, Porto Alegre, RS, Brasil. Tel.: (51) 3381.7976. E-mail: lisieux@telles.med.br

Não foram declarados conflitos de interesse associados à publicação deste artigo.

Copyright (c) Revista de Psiquiatria do Rio Grande do Sul - APRS

Recebido em 22/07/2010. Aceito em 17/12/2010 


\section{Introdução}

As agressões sofridas pelas equipes de saúde nos diferentes ambientes hospitalares têm prevalências muito variáveis $(0,4$ a $91 \%)$, sendo comum a falta de notificação desses $\operatorname{casos}^{1}$. Os profissionais envolvidos muitas vezes negam o risco de agressão; outra vezes, parecem aceitá-lo passivamente, como algo inerente à sua atividade ocupacional ${ }^{2}$.

Em hospitais psiquiátricos forenses, as consequências de comportamentos violentos de internos são graves, podendo variar de lesões leves a outros pacientes e equipe técnica, passando por danos materiais diversos e chegando até lesões severas ou mortes. Fatos como esses acarretam um prejuízo no tratamento do agressor, o qual, muitas vezes, tem seu regime terapêutico alterado, perde benefícios obtidos e pode permanecer por maior tempo em medida de segurança (MS). A falta de conhecimento sobre a incidência desse fenômeno e suas caracterísiticas favorece a perpetuação do problema.

Evidências recentes sugerem que alguns preditores de comportamento violento diferem de acordo com o tipo de transtorno mental, a situação e o tipo de conduta violenta. Por exemplo, estudos realizados com pacientes hospitalizados encontraram maior chance de conduta violenta entre portadores de síndrome cerebral orgânica, retardo mental e psicoses com leves sinais neurológicos. Na comunidade geral, os psicopatas representam o grupo com maiores taxas de crime violento ${ }^{3,4}$ e reincidem mais rapidamente quando comparados a grupos sem esse diagnóstico ${ }^{5}$.

Sabe-se que a não adesão aos psicofármacos e o uso de álcool e drogas constituem fatores associados ao fenômeno de múltiplas internações psiquiátricas ${ }^{6}$. Já as condutas violentas em portadores de transtorno mental são causa de hospitalização psiquiátrica e influenciam a evolução clínica e as intervenções terapêuticas, sendo necessário uma acurada avaliação de riscos. Em ambientes psiquiátricos forenses, além da violência, existem as atuações do tipo antissocial, contribuindo para modificar a periculosidade dos indivíduos e a evolução do tratamento.

Pesquisas sobre predição de conduta violenta entre pacientes hospitalizados possuem diferentes metodologias, assim como amostras de pacientes que variam consideravelmente quanto ao diagnóstico e à sintomatologia; algumas amostras relatam apenas quadros psicóticos, outras incluem pacientes com síndrome cerebral orgânica, demência, retardo mental e transtornos de personalidade.

Estudo de caso-controle com uma amostra de 137 pacientes psiquiátricos internados que cometeram 470 agressões evidenciou que, entre os internos violentos, houve predomínio de esquizofrênicos, com alucinações e hospitalizações psiquiátricas prévias ${ }^{7}$. A violência foi descrita como repetitiva e parte integrante de um padrão prévio de distúrbio de comportamento e conduta agressiva. Quanto à gravidade dos incidentes, houve predomínio de lesões de gravidade I (sem lesão detectável) em 59\%, seguido por 39\% de gravidade II (pequenas lesões, como arranhões ou pequenas lacerações) e $2 \%$ de gravidade III (grandes lacerações, fraturas, perda de consciência ou lesões que determinaram tratamento médico imediato). Ocorreram menos agressões a noite. As vítimas mais comuns foram enfermeiros e pacientes; médicos, outros integrantes da equipe e visitas foram menos frequentemente agredidos $^{7}$.

Os enfermeiros têm sido apontados como as vítimas mais comuns da violência perpetrada por pacientes psiquiátricos, como mostram um estudo israelense, um italiano e outro conduzido no Reino Unido ${ }^{8-10}$. O contato mais frequente entre a enfermagem e os pacientes, bem como o papel de cuidadores desses profissionais, pode contribuir para que eles sejam mais vitimados por pacientes hospitalizados. Já na comunidade, as vítimas mais frequentes dos doentes mentais são os familiares ${ }^{11}$. Psiquiatras sofrem violências físicas no início de suas carreiras, geralmente lesões menores, praticadas por pacientes psicóticos. O trabalho em emergências ou âmbitos forenses muitas vezes propricia mais de uma vitimização ${ }^{12}$. Estudo realizado em um grande hospital forense estatal estadunidense com o objetivo de determinar a taxa de psiquiatras agredidos por pacientes e avaliar as características desses profissionais revelou que $13 \%$ dos psiquiatras haviam sido feridos no hospital em um período de 5 anos $^{13}$. As características dos psiquiatras agredidos eram as seguintes: médicos mais jovens e com menos tempo de experiência profissional. Médicos do sexo masculino foram agredidos numa proporção em torno de $50 \%$ maior do que as do sexo feminino. Já a presença de aliança terapêutica positiva com o paciente parece diminuir o risco de violência hospitalar ${ }^{14}$

Pesquisa com população de pacientes psiquiátricos italianos baixados em unidade de agudos concluiu que as variáveis associadas a comportamentos violentos naquela população foram: idade menor do que 24 anos, sexo masculino, internação compulsória, presença de atitudes hostis no momento da admissão, portadores de esquizofrenia, transtorno afetivo, transtorno de personalidade, retardo mental, transtorno orgânico cerebral ou uso de álcool/drogas ${ }^{15}$. Outro estudo com pacientes esquizofrênicos internados encontrou como preditores de comportamento violento contra outras pessoas as variáveis sexo masculino, número de internações prévias e abuso de álcool ${ }^{16}$. $\mathrm{O}$ mesmo autor, em revisão posterior, concluiu que a única variável estática capaz de predizer violência em pacientes psiquiátricos hospitalizados era a ocorrência de agressão prévia, sendo as variáveis sexo, idade, diagnóstico e abuso de drogas de menor importância ${ }^{17}$.

Estudo americano de seguimento de pacientes psiquiátricos no período pós-alta verificou que o diagnóstico comórbido de abuso de substâncias foi um fator-chave na ocorrência de violência durante o período de 1 ano. Os índices de prevalência de violência variaram de $17,9 \%$ para pacientes com transtorno mental maior, $31,1 \%$ para pacientes com transtorno mental maior e abuso de substâncias e $43 \%$ para pacientes com outras doenças mentais e abuso de substâncias. As vítimas foram, em geral, familiares ou amigos dos pacientes $^{18}$. 
Entre a população de pacientes forenses hospitalizados, Ball et al. salientam como fatores preditivos de periculosidade a presença de comportamento grosseiramente impróprio (cuspir, limpar nariz em público), violência prévia, história de hospitalização psiquiátrica prévia e antecedente de 10 ou mais internações psiquiátricas ${ }^{19}$.

Em vista do exposto, os objetivos do presente estudo foram apresentar uma revisão bibliográfica atualizada do tema, informar a incidência de conduta violenta e antissocial em uma população manicomial e descrever a implementação de um método de registro sistematizado desse fenômeno.

\section{Método}

\section{Delineamento, população e local da pesquisa}

Estudo de coorte, prospectivo, realizado no período de maio de 2007 a maio de 2008, cuja população foi composta por 68 pacientes do sexo masculino em cumprimento de MS por crimes e diagnósticos diversos, hospitalizados na unidade D do Instituto Psiquiátrico Forense Maurício Cardoso (IPFMC). Foram excluídos do estudo pacientes que se encontravam usufruindo do benefício da alta progressiva, residindo fora da instituição.

O IPFMC é o único hospital psiquiátrico da Rede Penitenciária do Estado do Rio Grande do Sul, integrando a estrutura orgânica da Superintendência dos Serviços Penitenciários (SUSEPE) da Secretaria de Justiça e Segurança. A instituição cumpre funções periciais e assistenciais, além de atividades de ensino e pesquisa. O IPFMC é um dos 28 chamados hospitais de custódia e tratamento psiquiátrico da rede penitenciária nacional, sendo responsável pelo tratamento de todas as pessoas submetidas a MS no Rio Grande do Sul. A instituição também centraliza a realização de todas as perícias anuais para verificação da cessação de periculosidade.

\section{Instrumentos utilizados}

Dois instrumentos foram utilizados no presente estudo: a Escala de Agressividade Declarada de Yudofsky (Overt Aggression Scale, OAS) e o Questionário de Seguimento de Tengström et al., ambos em versões em língua portuguesa.

A OAS mede de forma objetiva o grau de comportamento agressivo em adultos e crianças, podendo ser usada para medir comportamento agressivo por um período de tempo prolongado ${ }^{20,21}$. Os comportamentos agressivos são divididos em quatro categorias, a saber: agressão verbal; agressão física contra objetos; agressão física contra si próprio; e agressão física contra pessoas. O item intervenção descreve possíveis condutas assumidas frente à(s) conduta(s) agressiva(s) e seus escores.

O outro instrumento, Questionário de Seguimento de Tengström et al., é destinado ao acompanhamento e identificação de conduta violenta e antissocial em populações de hospitais psiquiátricos forenses, consistindo em questionário para seguimento no período de $1 \mathrm{ano}^{4}$. As variáveis estudadas são as seguintes: agressão física contra a equipe terapêutica; agressão física contra pacientes; violência sexual; ameaças; e comportamento antissocial.

A recidiva de comportamentos foi observada durante 1 ano, sendo registrados episódios de conduta violenta e antissocial, codificados como recidiva em evento violento ou recidiva em qualquer evento.

\section{Aspectos éticos}

A pesquisa foi considerada de risco mínimo, por seu caráter observacional. $\mathrm{O}$ estudo foi aprovado pelo Comitê de Ética em Pesquisa do IPFMC e pela Carrera de Doutorado da Facultad de Ciencias Médicas da Universidade Nacional de La Plata (UNLP), La Plata, Argentina.

\section{Resultados}

As características sociodemográficas da amostra evidenciaram prevalência de brancos $(73,5 \%)$, solteiros $(79,4 \%)$, com baixa escolaridade (em média 3,74 anos), com ocupação à época do delito (58,8\%), idade média de 29,7 anos à época do delito e idade média atual de 42,8 anos.

Entre as variáveis clínicas estudadas, encontramos uma maior prevalência do diagnóstico de transtornos psicóticos $(58,8 \%$, englobando aqui todas as esquizofrenias e transtornos de humor com sintomas psicóticos), seguido de retardo mental (20,6\%), transtornos de personalidade $(14,7 \%)$ e dependência de álcool e/ou drogas $(5,9 \%)$.

O estudo das variáveis criminológicas evidenciou predomínio de conduta monodelitual entre a população de internos em estudo. Encontramos também maior prevalência de delitos contra a pessoa, tais como homicídio $(29,4 \%)$ e lesões corporais $(22,1 \%)$. Roubo e furto estiveram presentes em $20,6 \%$ da população, enquanto que outros delitos e delitos sexuais tiveram índices de 14,7 e 11,8\%, respectivamente.

Toda a população da coorte iniciou em oportunidade de recidivar em maio de 2007. No momento da censura administrativa, em maio de 2008, foram considerados os dados de seus respectivos tempos em oportunidade de recidivar para a análise dos resultados do seguimento. A média de tempo de ocorrência de recidiva em qualquer evento foi de 171,1 dias, e a mediana foi de 120 dias. Houve duas perdas por mortes em função de doenças clínicas, e $97,1 \%$ da população chegou ao término do estudo.

Durante o período de 1 ano, 200 eventos violentos ou antissociais foram registrados na OAS e no Questionário de Seguimento de Tengström et al., envolvendo 50 pacientes da amostra (73\%). A distribuição do número de eventos na população em estudo variou de 18 pacientes sem qualquer registro de intercorrências a um paciente com 21 registros de conduta violenta e antissocial. A média de eventos por paciente foi de 2,94 3 3,97. 
Todos os portadores de retardo mental apresentaram recidiva em qualquer evento, e $21,4 \%$ deles recidivaram em evento violento. Os portadores de transtorno de personalidade apresentaram a maior prevalência de eventos violentos quando comparados aos demais diagnósticos.

Os índices de recidiva violenta variaram de acordo com os resultados do juízo profissional de risco de recidiva violenta, sendo, respectivamente, de 4,5\% para risco baixo, $17,9 \%$ para risco médio e 38,9\% para risco alto. Esses achados apresentaram significância estatística $(\mathrm{p}=0,005)$.

Houve predomínio de conduta antissocial ou agressiva durante o dia $(73,5 \%)$. As intervenções mais prevalentes fo- ram o isolamento total (34\%), a conversa com o paciente (24\%) e o uso de medicação intramuscular (18\%). Em 18\% dos casos, não houve qualquer intervenção.

Dentre os registros na OAS, destaca-se a maior prevalência de agressão verbal $(57,1 \%)$ (Tabela 1$)$.

Na distribuição de eventos segundo o Questionário de Seguimento de Tengström et al., encontramos predomínio de condutas antissociais, tais como uso incorreto ou não uso de medicação $(19,5 \%)$, violação de regras institucionais $(17,1 \%)$, uso de droga ou álcool $(9,8 \%)$ e fuga da instituição $(9,8 \%)$. Entre as condutas violentas, destacam-se as agressões físicas contra pacientes sem necessidade de tratamento médico $(9,8 \%)$ (Tabela 2$)$.

Tabela 1 - Distribuição da amostra estudada segundo as categorias da OAS

\begin{tabular}{lcc}
\hline Categoria & Porcentagem & Porcentagem válida \\
\hline Agressão verbal & 17,6 & 57,1 \\
Agressão física contra objetos & 1,5 & 4,8 \\
Agressão física contra si mesmo & 1,5 & 4,8 \\
Agressão física contra pessoas & 10,3 & 33,3 \\
\hline
\end{tabular}

OAS $=$ Overt Aggression Scale.

Tabela 2 - Distribuição da amostra estudada segundo o Questionário de Seguimento de Tengström et al.

\begin{tabular}{lcc}
\hline Categoria & Porcentagem & Porcentagem válida \\
\hline Violência física contra equipe sntm & 2,9 & 4,9 \\
Violência física contra paciente sntm & 5,9 & 9,8 \\
Violência física contra paciente cntm & 1,5 & 2,4 \\
Intimidação sexual & 2,9 & 4,9 \\
Ameaça de violência & 2,9 & 4,9 \\
Comportamento ameaçador percebido como chantagem e ameaça & 1,5 & 2,4 \\
Dano a propriedade & 1,5 & 2,4 \\
Fuga da instituição & 5,9 & 9,8 \\
Furto & 4,4 & 7,3 \\
Uso de droga ou álcool & 5,9 & 9,8 \\
Tráfico de droga ou álcool & 2,9 & 4,9 \\
Violação de regras institucionais & 10,3 & 17,1 \\
Uso incorreto ou não uso medicação & 11,8 & 19,5 \\
\hline
\end{tabular}

$\mathrm{cntm}=$ com necessidade de tratamento médico; $\mathrm{sntm}=$ sem necessidade de tratamento médico.

\section{Discussão}

Na presente pesquisa, o estudo das variáveis sociodemográficas, clínicas e criminológicas evidenciou semelhança com estudos prévios sobre o tema ${ }^{22,23}$. No período de 1 ano, foram detectados 200 eventos violentos ou antissociais, envolvendo $50 \mathrm{pa}-$ cientes ( $73 \%$ da população estudada). Esse achado não confirma a crença de que há uma dificuldade envolvida em prever violência em pacientes hospitalizados, onde o tratamento institucional e medicamentoso poderia suprimir seriamente tal conduta ${ }^{24}$.

Os índices de recidiva violenta variaram de acordo com os resultados dos respectivos juízos profissionais, resultado que apresentou significância estatística. Tal achado ressalta a importância da avaliação clínica englobando aspectos da doença, do apoio familiar e de fatores socioambientais.

Os portadores de transtorno de personalidade apresentaram maior prevalência de eventos violentos quando comparados aos demais diagnósticos. Esse dado reafirma o conhecimento da maior periculosidade dessa população, mesmo dentro de uma instituição psiquiátrica forense.

Todos os pacientes com diagnóstico de retardo mental apresentaram recidiva em qualquer evento, e 21,4\% recidivaram em evento violento. Esse achado está de acordo com estudos realizados em populações psiquiátricas forenses americana 
e alemã, que mostraram que os comportamentos antissociais e as condutas ameaçadoras e/ou violentas foram bastante prevalentes nesse grupo diagnóstico ${ }^{4,25}$. Estudo de coorte em população sueca ressaltou o aumento de chance de violência quando o diagnóstico de retardo mental estava presente ${ }^{26}$.

O presente estudo possibilitou identificar o predomínio de eventos agressivos e antissociais durante o dia, o que está de acordo com a literatura ${ }^{7}$. Segundo os nossos registros na OAS, a conduta agressiva mais prevalente foi a verbal. Já entre as condutas antissociais mais registradas no Questionário de Seguimento de Tengström et al., salienta-se o uso incorreto ou não uso da medicação prescrita, as violações de regras institucionais, o uso de substância psicoativa e a fuga da instituição. As intervenções mais usadas, segundo os registros da OAS, foram o isolamento total, a conversa com o paciente e o uso de medicação intramuscular. Os cuidados terapêuticos e a contenção prestados em nível hospitalar podem ter influenciado a atenuação da gravidade de expressão de condutas violentas e antissociais.

Quanto às limitações do estudo, reconhece-se que o fato de os pacientes estarem hospitalizados e em tratamento, recebendo medidas para controle do risco de conduta violenta e/ou antissocial, pode ter representado um efeito protetor, minimizando a gravidade das intercorrências. Além disso, o estudo foi realizado em população exclusivamente masculina, permanecendo em aberto um caminho para investigações futuras em populações mistas e femininas. No entanto, apesar dessas limitações, acreditamos que o presente estudo propiciou uma visão mais fidedigna e menos preconceituosa dos riscos de conduta violenta e antissocial em populações psiquiátricas forenses. Foi possível também estabelecer um método sistemático de registro e avaliação de risco de violência, superando práticas tradicionais brasileiras anteriores.

\section{Conclusão}

O estudo introduziu um modelo sistemático de avaliação de risco de violência em população psiquiátrica forense brasileira, tornando a tarefa mais transparente, ética, dinâmica e em sintonia com a prática científica atual. Foi possível, também, pela primeira vez, medir a ocorrência de conduta violenta e antissocial nessa população.

Ao aprimorar as avaliações de risco de violência com o uso de instrumentos específicos para populações forenses, estar-se-á colaborando para a maior segurança da sociedade e das vítimas em potencial, bem como das pessoas passíveis de perpetrarem atos agressivos. Também se estará evitando possíveis erros de avaliação que possam privar da liberdade pessoas sem periculosidade ou minimizar futuros quadros de reincidência criminal.

Por ser esta pesquisa original no Brasil, acreditamos que será fonte de contribuição de dados na área. Finalmente, espera-se contribuir para o intercâmbio científico com outros centros de pesquisa, o que é uma proposta deste projeto desde o seu nascimento, ao integrar dois centros de investigação, a
Universidade Federal de Ciências da Saúde de Porto Alegre (UFCSPA) e a Facultad de Ciencias Médicas da Universidad Nacional de La Plata, a uma entidade do sistema prisional brasileiro.

\section{Referências}

1. Franz S, Zeh A, Schablon A, Kuhnert S, Nienhaus A. Aggression and violence against health care workers in Germany - a cross sectional retrospective survey. BMC Health Serv Res. 2010;10:51.

2. Abdalla-Filho E. Violência em saúde: quando o médico é o vulnerável. Bioetica. 2004;12(2):121-6.

3. Travin S, Lee HK, Bluestone H. Prevalence and characteristics of violent patients in a general hospital. J Med. 1990;90(12):591-5.

4. Tengström A, Hodgins S, Müller-Isberner R, Jöckel D, Freese R, Özokyay K, et al. Predicting violent and antisocial behavior in hospital using the HCR-20: the effect of diagnoses on predictive accuracy. Int J Forensic Ment Health. 2006;5:39-53.

5. Serin RC, Amos NL. The role of psychopathy in the assessment of dangerousness. Int J Law Psychiatry. 1995;18(2):231-8.

6. Haywood TW, Kravitz HM, Grossman LS, Cavanaugh JL, Davis JM, Lewis DA. Predicting the "revolving door" phenomenon among patients with schizophrenic, schizoaffective, and affective disorders. Am J Psychiatry. 1995;152:856-61.

7. Noble P, Rodger S. Violence by psychiatric in-patients. Br J Psychiatry. 1989;155:384-90.

8. Haim R, Rabinowitz J, Lereya J, Fenning S. Predictions made by psychiatrists and psychiatric nurses of violence by patients. Psychiatr Serv. 2002;53(5):622-4.

9. Winstanley S, Whittington R. Aggression towards health care staff in a UK general hospital: variation among professions and departments. J Clin Nurs. 2004;13(1):3-10.

10. Zampieron A, Galeazzo M, Turra S, Buja A. Perceived aggression towards nurses: study in two Italian health institutions. J Clin Nurs. 2010;19(15-16):232941. Epub 2010 Jun 14.

11. Binder RL. Are the mentally ill dangerous? J Am Acad Psychiatry Law. 1999;27(2):189-201.

12. Bloom JD. The character of danger in psychiatric practice: are the mentally ill dangerous? Bull Am Acad Psychiatry Law. 1989;17(3):241-55.

13. Carmel H, Hunter M. Psychiatrists injured by patient attack. Bull Am Acad Psychiatry Law. 1991;19(3):309-16.

14. Beauford JE, McNiel DE, Binder RL. Utility of the initial therapeutic alliance in evaluating patients' risk of violence. Am J Psychiatry. 1997;154:1272-6.

15. Biancosino B, Delmont S, Grassi L, Santone G, Preti A, Miglio R, et al. Violent behavior in acute psychiatric inpatients: a national survey in Italy. J Nerv Ment Dis. 2009;197(10):772-82.

16. Steinert $T$, Wiebe $C$, Gebhardt RP. Aggressive behavior against self and others among first-admission patients with schizophrenia. Psychiatr Serv. 1999;50(1):85-90

17. Steinert T. Prediction of inpatient violence. Acta Psychiatr Scand. 2002;106:133-41.

18. Steadman HJ, Mulvey EP, Monahan J, Robbins PC, Appelbaum PS, Grisso T, et al. Violence by people discharged from acute psychiatric inpatient facilities and by others in the same neighborhoods. Arch Gen Psychiatry. 1998;55:393-401.

19. Ball EM, Young D, Dotson LA, Brothers LT, Robbins D. Factors associated with dangerous behavior in forensic inpatients: results from a pilot study. Bull Am Acad Psychiatry Law. 1994;22(4):605-20

20. Yudofsky SC, Silver JM, Jackson W, Endicott J, Williams D. The Overt Aggression Scale for the objective rating of verbal and physical aggression. Am J Psychiatry. 1986;143:35-9.

21. Silver JM, Yudofsky SC. The Overt Aggression Scale: overview and guiding principles. J Neuropsychiatry Clin Neurosci. 1991;3(2):S22-9.

22. Folino JO, Urrutia MI. Mental disturbances and criminological characteristics in crime-accused insane as recorded at the judiciary office in La Plata, Argentina, for 10 years. Int J Law Psychiatry. 2001;24(4-5):411-26.

23. Telles LEB. Perícias de responsabilidade penal realizadas no instituto psiquiátrico forense. Multijuris. 2007;2(3):44-9.

24. Monahan J. Risk assessment of violence among the mentally disordered: generating useful knowledge. Int J Law Psychiatry. 1988;11:249-57.

25. Sreenivasan S, Kirkish P, Shoptaw S, Welsh RK, Ling W. Neuropsychological and diagnostic differences between recidivistically violent not criminally responsible and mentally ill prisoners. Int J Law Psychiatry. 2000;23(2):161-72.

26. Hodgins S. Mental disorder, intellectual deficiency, and crime. Arch Gen Psychiatry. 1992;49:476-82 